\title{
Enquête
}

Archives de la revue Enquête

3| 1996

Interpréter, Surinterpréter

\section{L'interprétation sauvage}

Wild interpretation

\section{Yannick Jaffré}

\section{(2) OpenEdition}

\section{Journals}

Édition électronique

URL : http://journals.openedition.org/enquete/513

DOI : 10.4000/enquete. 513

ISSN : 1953-809X

Éditeur :

Cercom, Éditions Parenthèses

Édition imprimée

Date de publication : 1 novembre 1996

Pagination : 177-190

Référence électronique

Yannick Jaffré, «L'interprétation sauvage », Enquête [En ligne], 3 | 1996, mis en ligne le 11 juillet 2013, consulté le 10 décembre 2020. URL : http://journals.openedition.org/enquete/513 ; DOI : https:// doi.org/10.4000/enquete.513

Ce document a été généré automatiquement le 10 décembre 2020. 


\title{
L'interprétation sauvage
}

\author{
Wild interpretation
}

\section{Yannick Jaffré}

Une lecture du livre de Tobie Nathan, L'influence qui guérit, Paris, Odile Jacob, 1994.

1 Les publications relevant de la psychiatrie transculturelle ont récemment connu en France un important développement qui, plus encore qu'à l'accroissement des flux migratoires et à la prise en considération de la détresse de certaines populations, correspond aux mutations des rapports à l'autre. Dans un premier temps, seul le psychiatre expatrié devait accorder son violon thérapeutique à des contextes exotiques. Nombre de recherches menées au Centre hospitalier de Fann à Dakar consistaient à adapter un savoir technique aux conditions locales d'exercice ${ }^{1}$. Les études se multiplièrent, englobant une réflexion sur les repères structuraux de la psychanalyse, l'utilisation des données culturelles dans les thérapies² ${ }^{2}$ ou l'aménagement de l'espace de soin. D'autres équipes se consacrèrent à une description anthropologique de cultes et rites de possession, et à l'étude des savoirs populaires étiologiques et thérapeutiques de la folie $^{3}$. La tension entre spécialisation de la psychiatrie et/ou secteur de l'anthropologie apparait ainsi comme constitutive de l'ethnopsychiatrie.

Cette obligation d'un double regard concerne maintenant des praticiens œuvrant dans leurs propres sociétés. Face à des patients venus d'ailleurs ou liés à d'autres appartenances, ils se trouvent dans une position singulière, rencontrant des difficultés pour établir leur diagnostic, et éprouvant le caractère souvent artificiel des cadres nosographiques qui leur sont familiers. À l'obligation de tenir compte des spécificités de pratiques de soins dans des situations de contact entre des groupes culturellement distincts, est supposée répondre la combinaison des études psychologiques et ethnosociologiques. L'anthropologie fournirait alors les grilles sémantiques et culturelles nécessaires à la compréhension d'une singularité subjective. La diversité des modalités de la «psychologisation du social » à l'oubli de l'individuel au profit d'un « tout social »qui peut ordonner cette mise en rapport de disciplines distinctes autour des rapports qu'un individu entretient avec les signifiants de sa culture, engage des questions d'interprétation. 
3 Dans le domaine français, un psychologue clinicien, Tobie Nathan, a publié plusieurs ouvrages d'ethnopsychiatrie clinique ${ }^{4}$ illustrant les liens tissés entre recherches cliniques et ethnologiques. Le dernier ouvrage de cet auteur, jouissant d'un fort succès médiatique, est précisément consacré à comprendre " comment la psychiatrie occidentale peut aider les populations immigrées qui ne partagent pas les mêmes références culturelles » et comment le médecin « peut s'inspirer de démarches traditionnelles pour vraiment aider des patients venus d'une autre culture ${ }^{5} »$. Pour brosser à grands traits le contexte sociopsychologique de sa pratique, T. Nathan utilise une série d'oppositions distinguant deux mondes: celui de la «pensée rationnelle» (p. 20), du «savoir objectif» (p. 21), de la « logique bien huilée » (p. 21), de « l'ordre intellectuel occidental» (p. 47), de « l'occident obtus» (p. 329), des «clergés et des syndicats de thérapeutes» (p.73), à celui des "pensées d'une densité inouïe» (p. 25), d'une "science plusieurs fois millénaire des guérisseurs » (p. 332) ${ }^{6}$. Au-delà de ce pathos idéologique ${ }^{7}$, ce texte peut permettre, à partir d'entretiens en situation pluriculturelle, d'ouvrir à une réflexion sur une interaction thérapeutique qui comprend, au minimum, deux opérations - comprendre et dialoguer - correspondant à deux problèmes distincts, celui du cadre interprétatif $\mathrm{du}$ discours du patient et celui du langage utilisé pour répondre à sa demande.

4 Face à la référence nosographique, ce n'est pas de manquer de systèmes d'interprétations dont souffre le «thérapeute transculturel», mais d'en trop posséder. Il peut en effet analyser le dire de son malade selon une grille nosographique « scientifique » ou selon les représentations "populaires » ou "savantes " de la société dont est originaire son patient. Très simplement, il peut choisir d'employer un métalangage interprétatif (psychologique, psychiatrique, etc.) ou utiliser une partie du code de son interlocuteur comme interprétant ${ }^{8}$ de son discours. Ces interrogations sont proches de celles rencontrées par l'ethnologue faisant alterner dans sa description données émiques et étiques. En fait, plus que des questions de différences culturelles, ces textes d'ethnopsychiatrie concernent des difficultés naissant de la mise en rapport d'une langue naturelle avec un code interprétatif artificiel ${ }^{9}$.

5 Pour des populations françaises, l'usage d'une même langue d'expression et la diffusion d'une certaine éducation sanitaire rend possible un dialogue médecin-patient à partir de quelques référents communs (représentation des parties du corps, postulats physiologiques et psychologiques simples, etc.). Selon les contextes, les problèmes se posent avec plus ou moins d'acuité ${ }^{10}$. Toutefois, de la "Corrèze au Zambèze », les questions sont les mêmes : chaque société, ou culture, face au discours médical (disease) communément utilisé comme référence, découpe différemment le continuum du pathologique en fonction de divers traits sémiologiques et étiologiques (illness) différenciant ainsi les expériences subjectives (sickness) de la maladie. Face à cette multiplicité d'interprétants potentiels, le choix du système thérapeutique de référence dépend, avant tout, des rapports institués entre médecine officielle et systèmes locaux d'interprétation ${ }^{11}$. Pour l'Afrique, est accepté un dialogue avec un ensemble de pratiques populaires de soins qui sont légalement exclues du champ de la thérapie en Europe.

Il s'agit aussi de légitimité scientifique ${ }^{12}$. Différant des autres «spécialités » médicales où les référents corporels (anatomopathologiques, biologiques, génétiques, etc.) offrent des points, même provisoires, de certitude, le discours psychiatrique se présente avant tout comme sémiologique. Il est donc soumis aux aléas d'hypothèses explicatives et de nouvelles catégorisations. Mais, hors le choix explicite d'un discours de référence, le thérapeute transculturel se trouve confronté à la simple succession des propos de ses 
patients. Il doit alors, «tout faire pour agir en Soninké avec un patient Soninké, en Bambara avec un Bambara, en Kabyle avec un Kabyle... » (p. 24), oscillant ainsi entre un œcuménisme identificatoire et un infini des interprétations. Le dialogue, quant à lui, implique à l'évidence que les interlocuteurs partagent un même code linguistique et sémiologique. Cette prise en compte du contexte est assez proche de la notion de « double ajustement réciproque » : des cadrages successifs sont imposés au soignant pour accorder son langage à celui de son patient. Pourtant, s'agissant d'une interaction à finalité thérapeutique, bien que nécessaire, cette adaptation culturelle n'est pas suffisante.

Dans la pratique biomédicale, le sens est construit par l'utilisation d'une terminologie scientifique ayant le corps et la maladie pour référents. Pour élaborer son diagnostic, le soignant tente de réduire la polysémie des termes utilisés par le malade en les ramenant à leur référent corporel. Il transforme alors les symptômes évoqués en signes cliniques d'une réalité physiologique ${ }^{13}$. Les paroles du malade sont ainsi confrontées à la "vérité objective » de la maladie. Autrement dit, le signe est conçu comme le représentant de la maladie et non comme le signifiant d'un sujet. La psychanalyse utilise d'autres critères et insiste sur l'ensemble des réseaux associatifs liés au signe identifié ${ }^{14}$. À l'envers de "l'ordre médical », elle souligne que les symptômes évoquent le sujet à son insu ${ }^{15}$. Dans ce cadre, les paroles ne peuvent être fausses ; mais simplement vides dès lors qu'elles se bornent à reprendre le discours commun et ne sont attribuables à aucun sujet.

En fait, en ethnopsychanalyse, l'interprétation concerne deux niveaux distincts. Il faut d'abord définir les conceptions du normal et du pathologique dans une société donnée, puis utiliser ces connaissances pour permettre à un patient d'accéder au sens de sa souffrance ${ }^{16}$. Dans ce domaine, le choix des procédures employées pour articuler le social et le singulier apparaît donc comme essentiel. Faute de précautions méthodologiques, le rapport à l'autre risque de se présenter sous les traits d'une " interprétation sauvage »interprétation qui ne résulte pas de la cohérence des données mais de l'arbitraire de l'interprète, même si cet arbitraire se dissimule sous une apparente cohérence rhétorique - dont il est possible de souligner quelques caractéristiques.

\section{L'équivalence et la comparaison}

Une des manières de définir un signe est d'en comparer la signification avec celle d'autres termes appartenant à des champs sémantiques proches ${ }^{17}$. Évoquant plusieurs cultures, T. Nathan commente ainsi des notions appartenant à des aires culturelles différentes. Par exemple la « frayeur » est supposée équivalente à « diatigè » en bambara et « sama fit dem nat» en wolof, puisque tous ces sentiments signifieraient "l'extraction du noyau du sujet », ce dernier étant lui-même équivalent à " âme en arabe, ro' $h$; double en bambara, dia; principe vital en wolof, fit» (p. 225). Ce procédé rhétorique de mise en abîme est fréquemment utilisé par l'auteur. Un autre texte propose, par exemple, une « définition ethnopsychanalytique » de la possession, qui prend la forme d'une énumération de divers cultes - Loa haïtien, Zar éthiopien, Lup serer, Tromba malgache, etc.; elle signifie globalement «que la pensée prend le corps », tout en jouant, parfois, comme pour le Loa ou « au Niger» (sic), d'une "ambiguïté » puisque «le cavalier est au-dessus (donc masculin), mais il écarte les jambes (il est donc féminin) ${ }^{18}$ ».

10 Ce procédé suscite bien des réserves. Les premières concernent le sens accordé aux termes utilisés. À suivre les travaux de Zempléni ${ }^{19}$, le fit (énergie, force vitale) et le roo (souffle qui quitte le corps au moment de la mort) en wolof seraient équivalents au $n i$ 
bambara (souffle et énergie vitale), en revanche le takandeer (ombre du corps humain) serait le dia (ombre). Or, le bambara en distingue plusieurs types : le ja naloma (lit, ombre stupide), le janin kegun (ombre maligne), etc., cette dernière quittant le corps au moment du décès... On l'aura compris, comparaison n'est pas équivalence: les champs sémantiques ne sont pas isomorphes. Quant au second exemple, à ces réserves s'ajoutent diverses contradictions avec des données émiques ${ }^{20}$. À l'évidence, on ne peut résoudre le problème du comparatisme ni par de hâtives équivalences ni par une "psychiatrisation " du social.

11 Comparer signifie définir des similitudes et des différences entre divers objets, ce qui suppose qu'ils puissent être soumis à un même ordre de mesure, c'est-à-dire qu'ils possèdent (ou qu'on leur attribue) un ensemble de dimensions communes ou qu'ils appartiennent à un même ordre de phénomènes. Dans ce domaine ethnopsychiatrique, trois « entrées » peuvent ouvrir à une démarche comparative. Il est tout d'abord possible de travailler sur des référents semblables. Si la question d'un instrument de mesure, notamment épidémiologique ${ }^{21}$ est supposée résolue, l'interprétation s'attachera non pas à homogénéiser les données, mais à souligner que semblable ne veut pas dire identique : il s'agit de comprendre comment la diversité des contextes détermine des représentations, des usages ou des appropriations variables d'objets identiques. Inversement, cette question peut, en second lieu, être posée à partir des interprétations et des discours locaux. Ainsi, diverses populations semblent s'accorder sur les mêmes représentations, notamment étiologiques, de la folie. Il s'agit ici, non pas d'homogénéiser des conceptions et des pratiques - comme celle de la possession - mais d'en évaluer la valeur dans divers contextes précis avant de les comparer. Enfin, les populations se côtoient, échangent leurs savoirs et traduisent spontanément leurs souffrances. "Justes » ou "fausses », ces traductions montrent que des acteurs en situation «dialogique» établissent quotidiennement des équivalences entre des sensations et des pathologies. Il sera toutefois nécessaire de distinguer entre ces diverses relations langagières (locuteurs plurilingues, dialogue entre malades, entre soignants et malades, usage commun de néologismes, etc.). Sinon, faute de se fixer des règles et de les énoncer clairement, une recherche comparative risque fort de n'être qu'une accumulation où « tout est dans tout, et inversement $»$.

\section{La métaphore au réel}

T. Nathan insiste, à juste titre, sur l'importance qu'il convient d'accorder à la langue de ses interlocuteurs - « leur langue maternelle, à partir de leurs propres théories » (p. 331). Quelques termes autochtones fleurissent en effet dans le texte, notamment en langue bambara ; leur utilisation pourtant ne va pas sans poser quelques problèmes ${ }^{22}$.

Les métaphores, parfois commentées dans le seul sens symbolique, sont « durcies ». Ainsi, pour reprendre un exemple précédent, que ja signifie le « double » de la personne, et tigè " couper ", n'implique pas qu'une personne disant $n$ ja tigéra (litt. mon double/âme est coupé) exprime "l'extraction du sujet ou plutôt du noyau du sujet » (p. 225). Depuis César et Pagnol nous savons que «fendre le cœur " n'est pas automatiquement une cause de décès. Bref, admettre que le signifiant puisse parfois s'exprimer, pour un sujet, sous la forme d'un symptôme somatique ne signifie pas que toute catachrèse soit à prendre « au pied de la lettre ${ }^{23}$ ". 
Ces locutions, souvent des mémoriaux de croyances anciennes, sont, de plus, liées à une histoire sociolinguistique. Leur existence actuelle n'atteste donc pas automatiquement qu'un sujet contemporain adhère aux idées qu'elles véhiculent. Dans un contexte français, les locutions «se faire du mauvais sang, ou de la bile" s'accordent avec l'ancienne théorie des humeurs, mais elles ne permettent pas d'inférer que des acteurs sociaux attribuent leur désarroi à leur vésicule, alors que c'est ce que laisse supposer T. Nathan en pays bambara : « La colère comme la frayeur est [...] articulée à une notion de membrane interne qui cède sous l'effet d'une intense pression. Dans la frayeur, c'est la coquille de l'âme qui, s'ouvrant accidentellement, laisse échapper le noyau de la personne; dans la colère, c'est la vésicule contenant la bile qui crève, permettant à la substance amère (en arabe, la bile se dit marara "amertume") de se répandre à travers le corps, peut-être même de cheminer jusqu'à la tête, provoquant la fureur. » (p. 246). Qu'un patient en pays bambara exprime sa souffrance en disant « $n$ kono gwanen don » (litt. mon ventre est chaud) ne signifie ni qu'il partage une conception de la maladie opposant le chaud au froid, ni qu'il s'apprête à prendre sa température.

\section{Traduction et transposition}

15 Il est enfin impossible d'évaluer la valeur d'un terme hors de son champ sémantique : une interprétation doit d'abord être textuellement cohérente avant de pouvoir être utilisée pour décrire une situation ou servir de base à une traduction. Ainsi la notion d'inquiétude (angoisse et/ou frayeur) ne peut être décrite que par approximations fines opposant par exemple le «trop» calme (suma) au ressassement (miiri), la nostalgie (nyènafin), les "problèmes» (kono gwan), la détresse (dusu kasi), etc. Privée d'un préalable ethnolinguistique - une opposition symbolique ne peut être directement liée à une interprétation sans être au préalable située dans une organisation dont elle ne constitue qu'un élément -, la traduction n'est plus qu'une transposition ne permettant ni de rendre compte de l'évolution des sentiments vécus par un patient ${ }^{24}$, ni d'entreprendre un travail comparatif entre diverses cultures et populations.

\section{La faiblesse des inférences}

16 A minima, l'interprétation consiste à établir des relations entre des faits et des discours supposés en apporter une explication. Dans la cure ethnopsychanalytique s'entrecroisent des propos de malades et des propositions relevant de l'anthropologie et de la psychologie. Il serait présomptueux de s'interroger ici sur la « véridicité » des diverses disciplines utilisées. En revanche, il est possible de mettre en relation des énoncés des patients avec une interprétation du thérapeute. Reprenons ici un exemple choisi par T. Nathan. Un homme de nationalité mauritanienne et d'origine soninkée, vit douloureusement un divorce qui le confronte à des règles différentes selon sa société ou la juridiction française ${ }^{25}$. Il décrit sa situation en utilisant un proverbe - «La tortue ne quitte pas sa carapace!»- interprété par le thérapeute comme signifiant « lorsqu'on vit au milieu d'étrangers, on ne montre pas sa nature, on se tient tranquille", " on ne commet pas l'imprudence de révéler à un étranger sa véritable pensée » (p. 23). L'analyste répond alors par un proverbe bambara - tu aperçois une calebasse de belle apparence descendant le Niger, c'est que son fond est troué -, ayant selon lui pour effet de permettre à son patient « d'être avant tout soninké (la seule façon d'être humain pour un Soninké) 
et d'évoquer, à la façon soninké, ses griefs envers sa femme ». Le patient propose à son tour comme réponse : «Si tu trouves une bicyclette dans le fleuve, saches que tu devras venir la déposer à l'endroit où tu l'as prise!» Cet échange est ainsi commenté par le thérapeute : «Avais-je fait autre chose que lui signifier : tu as désiré une femme jeune et jolie ; qui sait si elle n'appartenait pas à quelque génie du fleuve?» Outre des questions linguistiques et anthropologiques mal maîtrisées, comme l'assimilation rapide entre le bambara appartenant au groupe linguistique mandingue et le soninké appartenant au groupe soudanien, alors qu'il n'y a pas d'intercompréhension entre leurs langues, cette interaction langagière ne va pas sans poser problème. Les premiers portent sur le sens commun. Il faut bien accepter qu'une interprétation obéisse à certaines contraintes, et tienne compte d'une acception première, celle "qu'accepterait une communauté d'interprètes visant à un accord quelconque, sinon sur les meilleures interprétations, du moins sur le refus de celles qui sont indéfendables ${ }^{26} »$. Il semble normal que, dans un contexte de séparation, le patient veuille signifier qu'une femme prise chez ses parents doit être ramenée chez eux. Quant au dicton utilisé par le thérapeute, il souligne fort banalement qu'il faut se méfier des apparences. Cette idée est largement illustrée en pays bambara : «Si tu vois un barbican insulter le père d'un héron, c'est que son logeur est une autruche ", ou "il ne faut pas choisir sa femme un jour de fête», etc. Ces sentences invitent à se méfier d'une prétendue faiblesse dissimulant d'importantes relations ou à prendre garde aux charmes des atours féminins. Ainsi, dans cet entretien, rien n'autorise à supposer l'existence d'un " génie ». L'absence de relations cohérentes entre les diverses données textuelles du dialogue rend donc peu plausible une telle interprétation.

\section{L'univocité des causes}

17 Les assertions du thérapeute, hypostasiant quelques données, supposées heuristiques d'une situation complexe, relèvent globalement d'une option culturaliste. Ce choix n'est pas sans conséquences. Ainsi, pour reprendre notre exemple précédent, soulignons que cette interprétation laisse " un reste »: des enfants et une femme, "qui, influencée par les assistantes sociales et les puéricultures de la PMI, a demandé le divorce selon la loi française ». La question est reprise plus avant: ce sont les travailleurs sociaux qui, « au nom d'une idéologie sans âme et à force d'arguments et de philosophie sommaire ", auraient incité une femme bambara venue se plaindre de son mari à « divorcer et à tout entreprendre pour obtenir la garde des enfants. Savent-ils seulement qu'on est bambara par son père? Que les enfants appartiennent à l'ancêtre du patrilignage?» (p. 330). À l'évidence, l'analyse ignore les débats maliens concernant le code de la famille, le taux élevé de divorces et de naissances prématrimoniales à Bamako... Retenons ici que cette relation thérapeutique laisse apparaitre des patients-acteurs utilisant plusieurs univers sociaux concurrents et négociant une nouvelle définition des rapports entre hommes et femmes où le désir ne serait plus assujetti à une unique fonction reproductrice ${ }^{27}$. Expérience souvent difficile, l'émigration est aussi une occasion de définir d'autres relations de subordination et d'obtenir d'autres droits. Il ne s'agit donc pas que de croyances et, si l'on peut s'accorder sur la notion d'une autonomie des interprétations causales par un patient, il serait absurde d'imaginer une indépendance totale du trouble par rapport à ses déterminants sociaux. Dans ces rapports qui lient des coutumes et une nouvelle définition des rôles de chacun ${ }^{28}$, les enjeux concernent le présent, comme le 
souligne un proverbe bambara : «Le lièvre d'aujourd'hui, c'est le chien d'aujourd'hui qui le chasse.»

\section{La preuve par l'effet et l'univocité de l'interprétation} effets : «Lorsque je raconte une histoire à quelqu'un, c'est à la modification de son état que je perçois que l'histoire a été reçue.»(p.140). Si cette attention à la valeur performative du langage est compréhensible dans un cadre thérapeutique, elle laisse cependant entière la question de l'adéquation entre interprétation et dire du patient.

(un thérapeute et des cothérapeutes) incite au transfert ou tout au moins à la croyance, comme en témoigne cette malade qui déclare à propos de T.Nathan: "Ça fait des mois que son djinna me parle dans les oreilles." (p. 135). Or, dès lors qu'on recherche une «modification durable » de l'état des patients, la question « ça marche? ? est fondamentale ${ }^{29}$. Toutefois l'écart entre des interprétations ponctuelles, strictement culturelles, et la complexité des demandes des patients incite à s'interroger sur la durée bienfaisante de telles injonctions. Deux séries de questions concernent ici les modalités de l'interprétation et son adéquation aux situations vécues. Ainsi dans un cas (p.136), les troubles d'une patiente délirante et errante sont univoquement interprétés en fonction d'une grossesse gémellaire. Pour s'en tenir au matériau présenté, soulignons, outre une situation socio-économique et familiale complexe, que d'autres éléments probablement signifiants ne sont pas commentés. Ainsi le prénom de la patiente, tèné, est le même que celui de sa tante paternelle; il lui fut sans doute attribué comme marque d'attachement de son père envers sa propre sœur ou comme consolation de sa stérilité. De même des colas sont présentées par la malade, à la suite d'une demande du thérapeute, et utilisées lors de la cure. Sont-ce des yèlè woro évoquant la chance, filanin woro pour les jumeaux, sokun woro pour le pouvoir? Ces quelques signifiants, au hasard de notre lecture, évoquent ainsi la parenté, la reproduction, une possible "emprise » d'une tante... Il est donc fort peu probable que cette pathologie se laisse réduire à une seule causalité et résoudre par une seule réponse. Loin d'une interprétation univoque et ponctuelle, la situation de désordre doit être appréhendée globalement, d'autant plus qu'un éventuel pacte noué avec un génie devra être honoré, dans le temps, sous peine de " représailles ${ }^{30}$ ».

\section{Les figures de l'autre}

Il reste à comprendre la situation d'un malade vivant de manière syncrétique ces langages des sens multiples où s'enchâssent anciennes conceptions et obligations d'une société d'accueil. Dans les cures présentées, les patients apparaissent comme exemplaires de leurs origines « ethniques ", et l'acte thérapeutique est décrit comme une corrélation "d'univers complémentaires entre des sensations physiques et des univers culturels " (p.137). Or, il est hasardeux de ne définir le contexte social du patient que par quelques croyances supposées unanimement partagées ${ }^{31}$.

21 Cette définition d'une identité accordant globalement le singulier au commun a été naguère abordée par Jean Bazin, à propos d'un éventuel critère de «bambaraiité32 ». Avec sagacité, il remarquait que « l'ethnie n'est jamais, en fait, un simple cadre formel dont la 
commodité opératoire compenserait l'arbitraire. Elle tient toujours la place d'un sujet auquel nous reconnaissons au moins assez d'existence pour pouvoir lui attribuer comme prédicats des énoncés, des événements, des rapports sociaux dont nous pourrions donner une autre description si nous étions libérés de cette référence obligée ». Force est de reconnaitre que les cures présentées ici tombent sous cette critique. Faute de référents empiriques, le thérapeute tient plus un discours sur les autres qu'il n'appréhende le discours des autres ${ }^{33}$. L'anthropologue soulignera que c'est bien légèrement faire fi de l'hétérogénéité des groupes sociaux constituant les sociétés africaines contemporaines et négliger la pluralité des recours et des systèmes d'interprétations. Le psychologue soupçonnera qu'il s'agit de proposer ${ }^{34}$ au patient une identification imaginaire de plus, dont la spécificité ne serait que d'être une méconnaissance « ethnique ».

\section{Le recours et le retour}

Les notions utilisées - guérisseurs traditionnels, possession, génies... - amalgament des pratiques d'une telle diversité qu'elles semblent peu adaptées à circonscrire et décrire leur objet. Reste, malgré tout, l'importance des questions traitées puisque ces " autres ", même en se limitant à l'émigration malienne en France, représentent au moins 60000 personnes ${ }^{35}$. Les multiples difficultés rencontrées par ces populations concernent tant les différences entre les règles sociales du pays d'origine et celles du pays "d'accueil » (religion, polygamie, nombre et éducation des enfants, scolarisation, compétences et clivages linguistiques...) que les jeux de pouvoirs entre communautés locales et émigrées (atténuation du système des castes, du pouvoir des aînés, retour des enfants, etc.) ${ }^{36}$. Toutefois, quoique difficiles, voire douloureuses, ces transactions entre le propre et l'emprunté ne peuvent être conçues sous la forme pathétique de la domination - «je l'affirme haut et fort, les enfants des Soninkés, des Bambaras, des Peuls, des Dioulas, des Ewoundous, des Dwalas, que sais-je encore ? appartiennent à leurs ancêtres. Leur laver le cerveau pour en faire des blancs, républicains, rationalistes et athées, c'est tout simplement un acte de guerre.» (p. 331) - ou de la préservation - «Dans les sociétés à forte émigration, il faut favoriser les ghettos - oui, je le dis haut et clair -, favoriser les ghettos afin de ne jamais contraindre une famille à abandonner son système culturel. » (p. 216). Cet argumentaire du «bon cœur» risque en effet de déloger de leurs vies les objets mêmes d'une telle sollicitude. L'histoire de ces acteurs témoigne en effet d'autres choix, et d'une autre vitalité. Ainsi quatre cents associations regroupant des populations sénégalaises, maliennes et mauritaniennes ont été dénombrées en France en 1991 et, pour se limiter au secteur de la santé, durant cette même année, soixante-dix projets, représentant un financement de onze millions de francs, ont été mis en œuvre. Bref, il ne s'agit pas ici de nostalgie culturelle mais de dynamiques sociales, "d'interface entre les communautés plus ou moins organisées, les pouvoirs publics et les institutions locales ${ }^{37}$ ».

On admet aisément que la prise en charge d'un patient oblige à considérer simultanément des questions psychologiques et des données sociales ${ }^{38}$. Mais ces dernières, qui ne se limitent pas à quelques symboles, doivent inclure les acteurs sociaux, individuels ou collectifs, leurs stratégies, leurs enjeux... Seules de telles études permettraient de rendre compte des multiples rationalités qui traversent les sociétés africaines, dont celles constituées autour de l'émigration. Sous l'apparente uniformité de l'étranger, diverses situations se rencontrent: entre un enfant africain élevé en France, des femmes pratiquant de nouvelles formes de noria, ou des travailleurs provisoirement expatriés, le 
seul point commun est peut-être d'avoir « un double horizon» comme référence. Cet « ailleurs » diffère cependant d'un groupe à l'autre, constituant autant de « communautés narratives ${ }^{39}$ » articulant diversement l'inscription en un lieu et l'adaptation à une histoire ${ }^{40}$. Gageons que l'adoption d'une telle problématique pourrait aussi influer sur la prise en charge des patients. Mais il s'agit là d'une autre histoire où, l'originaire, dans la cure, ne serait plus un retour au guérisseur, mais un recours pour vivre le présent dans sa complexité et non dans des ghettos.

\section{NOTES}

1. H. Collomb, « Rencontre de deux systèmes de soins. À propos de thérapeutiques des maladies mentales en Afrique », Social Science and Medicine, VII, 1973, p. 623-633.

2. M.-C. et E. Ortigues, EEdipe africain, Paris, Plon, 1966 ; M.-C. Ortigues, P. Martino, H. Collomb, "L'utilisation des données culturelles dans un cas de bouffée délirante», Psychopathologie africaine, III, 1967, p. 121-147.

3. A. Zempléni, «La dimension thérapeutique du culte des Rab. Ndop, Tuuru et Samp, rites de possession chez les Lebou et les Wolof », Psychopathologie africaine, II, 1966, p. 295-439 ; A. Adler et A. Zempléni, Le bâton de l'aveugle, Paris, Hermann, 1972.

4. La folie des autres. Traité d'ethnopsychiatrie clinique, Paris, Dunod, 1986 ; Le sperme du diable, Paris, Presses universitaires de France, 1988.

5. L'influence qui guérit, Paris, O. Jacob, 1994 (quatrième de couverture).

6. II s'agit ainsi de protéger « un patient migrant de tout acte thérapeutique s'appuyant sur une causalité de type scientifique [qui] constitue à lui seul un nouveau traumatisme psychique » (p. 22), pour éviter qu'une fois «adultes, ces enfants noirs élevés à la française [ne deviennent] les plus insipides de tous les blancs » (p. 331).

7. Qui, parfois, n'est pas sans conséquences sanitaires : A. Prual, «Traditional Uvulectomy in Niger. A Public Health Problem ? ", Social Science and Medicine, XXXIX, 1994, p. 1077-1082, montre, à partir d'une enquête épidémiologique, que de sévères complications médicales nécessitant hospitalisation sont dues à une pratique populaire d'ablation de la luette en pays zarma et haoussa.

8. «Un métalangage critique n'est pas un langage différent du langage objet. C'est une portion du même langage objet et, en ce sens, c'est une fonction que n'importe quel langage remplit lorsqu'il parle de lui-même », U. Eco, Les limites de l'interprétation, Paris, Grasset, 1992, p. 44.

9. Ce langage interprétatif artificiel emprunte des termes aux langues naturelles, les vide de leurs signifiés puis les emploie pour expliquer les signifiés d'autres termes. Cette opération est assortie de diverses contraintes. Ainsi selon A. J. Greimas, Du sens, Paris, Seuil, 1970, p. 30, une taxinomie scientifique est convenable si elle est exhaustive et cohérente, "c'est-à-dire que chaque terme objet ne puisse être placé qu'à un seul endroit de l'arbre taxinomique et non à plusieurs »; il faut qu'elle « soit simple, et enfin qu'elle présente la classification sous sa forme la plus économique ». 10. Ce qui ne veut pas dire que la maladie ou le désordre ne soient pas, dans le domaine français, diversement connotés; cf. S. Fainzang, "L'alcool, les nerfs, le cerveau et le sang ", L'Homme, XXXV (135), 1995, p. 109-125.

11. J.-P. Dozon, «Ce que valoriser la médecine traditionnelle veut dire », Politique africaine, 28, 1987, p. 9-21. 
12. Cette question est traitée radicalement par T. Nathan : «Je ne suis pas loin de penser que la psychologie [...] serait une pure fiction » (p. 25).

13. Cf. R. Barthes, "Sémiologie et médecine", in L'aventure sémiologique, Paris, Seuil, 1985, p. 273-283.

14. Cette posture engage aussi des questions de "pouvoir médical ", puisque c'est admettre que le patient n'est pas sans savoir sur sa maladie. Sur ce point cf. notamment G. Raimbault, Médecins d'enfants (onze pédiatres, une psychanalyste), Paris, Seuil, 1973.

15. J. Lacan, "Fonction et champ de la parole et du langage en psychanalyse ", in Écrits, Paris, Seuil, 1971 : « Pour libérer la parole du sujet, nous l'introduisons au langage de son désir, c'est-àdire au langage premier dans lequel, au-delà de ce qu'il nous dit de lui, déjà il nous parle à son insu, et dans les symboles du symptôme tout d'abord » (p. 293).

16. Sur la différence entre le ressenti de la souffrance et la construction médicale de la douleur, I. Baszanger, Douleur et médecine, la fin d'un oubli, Paris, Seuil, 1995.

17. U. Eco, Sémiotique et philosophie du langage, Paris, Presses universitaires de France, 1984 : "Naturellement, dans une sémantique à interprétants, chaque interprétation est à son tour sujette à interprétation » (p. 110).

18. T. Nathan, La folie des autres, traité d'ethno-psychiatrie clinique, Paris, Dunod, 1986, p. 144-147.

19. A. Zempléni, op. cit., p. 310.

20. Cf. J.-P. Olivier de Sardan, "Possession, affliction et folie : les ruses de la thérapisation", L'Homme, XXXIV (131), 1994, p. 8 : « Il s'agit là d'une interprétation non fondée empiriquement, et en contradiction avec les représentations autochtones, émiques. Disons-le abruptement (nous nuancerons plus loin) : pour les adeptes de la plupart des cultes de possession africains et afroaméricains, il n'est aucunement question de soigner de la maladie mentale » (p. 8).

21. «Dans la science médicale, le niveau «maladie » prévoit, par définition, la connaissance de l'étiopathogenèse, du traitement et de l'évolution. [...] Dans le domaine de la psychiatrie, il n'y a pas d'accord entre les différentes écoles sur l'étiopathologie, le traitement et le pronostic d'une grande partie de ces affections. On travaille donc sur du matériel flou », P. Coppo, « Problèmes et limites méthodologiques des études épidémiologiques en situations transculturelles", Psychopathologie africaine, XXIII, 1990-1991, p. 279-285.

22. Sans nous attarder sur des fautes de transcription, quoique des règles existent pourtant depuis 1967 (diatigè au lieu de jatigè ; subakha au lieu de subaga, etc.), soulignons les erreurs : diabatigè (terme pourtant « savamment » commenté p. 224) n'existe pas en langue bambara, et ne pourrait s'entendre que comme « oignon / couper ». Le terme exact serait jatigèba, lexicalement correct mais inusité ; le terme tigè-tigè, lui aussi commenté, signifie fendre ou couper, mais jamais scarifier dont la traduction est $c i$, etc.

23. Ce dont témoignent, bien évidemment, les travaux de Freud sur l'hystérie : cf. «Fragment d'une analyse d'hystérie (Dora)», in Cinq psychanalyses, Paris, Presses universitaires de France, 1970.

24. Cf. P. Coppo, ed., Essai de psychopathologie dogon, Pérouse, CRMT / PSMTM, s.d.

25. Sur la complexité des procédures de divorce entre normes juridiques multiples, D. Kintz, «L'amant blessé ou une discussion peule sur le pluralisme juridique », Politique africaine, 40, 1990, p. 42-50.

26. U. Eco, op. cit., p. 14, 17. Eco ajoute : « Même le déconstructiviste le plus radical accepte l'idée qu'il y a des interprétations scandaleusement inacceptables. Cela signifie que le texte interprété impose des restrictions à ses interprètes. Les limites de l'interprétation coïncident avec les droits du texte (ce qui ne veut pas dire qu'ils coïncident avec les droits de son auteur). »

27. Pour une analyse des difficultés sexuelles ressenties par des femmes maliennes, cf. D. LutzFuchs, Psychothérapies de femmes africaines, Paris, L'Harmattan, 1994. 
28. Cette adaptation des règles aux nouvelles situations est incluse dans la notion même de « coutume»: E. Le Roy, «Les usages politiques du droit», in C. Coulon et D. C. Martin, eds, Les Afriques politiques, Paris, La Découverte, 1991.

29. Cf. A. Zempléni, « Où en est l'anthropologie médicale appliquée », in AMADES, 1992, p. 27-28.

30. En milieu mandingue, cf. J.-M. Gibbal, Tambours d'eau, Paris, Le Sycomore, 1982, p. 240 : «En matière de maladie mentale, j'ai pu observer une nette amélioration auprès de quelques patients, arrivés chez le jinétigi dans un état de prostration complète. Mais dans bien des cas c'est seulement une socialisation et une régulation des troubles qui résultent de la cure. Le patient devient époux ou épouse du génie dont la présence dans sa vie explique la déviance. »

31. Par exemple: "Mon interprétation terminale reprend une cosmogonie très répandue en Afrique [...] selon laquelle le monde s'est peuplé à partir d'un couple de jumeaux primordiaux. » (p. 137) ; «Un père qui tombe devant son fils, c'est comme si le monde s'écroulait. En Afrique il n'y a qu'une solution... » (p. 190).

32. J. Bazin, "À chacun son Bambara », in J.-L. Amselle et E. M'bokolo, eds, Au cœur de l'ethnie, Paris, La Découverte, 1985, p. 87-127.

33. J.-P. Olivier de Sardan, « Le réel des autres », Cahiers d'Études africaines, XXIX, 1989, p. 127-135. 34. «Proposer" est un euphémisme si l'on en juge par certains dialogues (p. 136) : "Après plusieurs minutes de contemplations, j'énonce (T. N.) : "Il s'agit d'une histoire de jumeaux!" Téné demande: "Veux-tu dire que dans mon ventre-là, je porte un couple de jumeaux ?" Je répète: "Non! À l'origine il s'agit d'une histoire de jumeaux!” Téné demande encore: "Ma voisine vient d'accoucher de deux jumeaux. Peut-être est-ce ceux-là que tu viens de voir ?" " 35. S. Diarra et M. Nadio, Migration et urbanisation au Mali, Bamako, CERPOD, I (7), 1993.

36. C. Quiminal, Gens d'ici, gens d'ailleurs, Paris, C. Bourgois, 1991. Soulignons que d'un point de vue de santé publique, il ne serait pas inutile de posséder des données d'épidémiologie descriptive sur la santé mentale de ces populations avant de préconiser des « solutions ».

37. M. Cissé, «Un pari : créer un centre de santé », Hommes \& Migrations, 1165, mai 1993, p. 23, 30-32. L'auteur, animateur local d'une association de migrants, déclare par exemple : « Avoir un personnel performant est d'autant plus important que les gens vont d'abord voir les médecins traditionnels et qu'en cas d'échec, ensuite, ils se rendent au centre de santé ».

38. Nous retrouvons ici l'enthousiasme de J. Berque, Mémoire des deux rives, Paris, Seuil, 1989, p. 276 : «Deux sociétés se recouvrant sur le même sol s'intériorisent l'une à l'autre, de sorte qu'il n'est plus entre elles d'autre solution qu'impliquant une réciprocité. Je ne parlerais plus d'immigration mais, risquons le néologisme, d'enmigration : voyage vers soi-même en l'autre, et vers l'autre en soi. "

39. Cf. J.-B. Metz, La foi dans l'histoire et dans la société. Essai de théologie fondamentale pratique, Paris, Cerf, 1979. Cette notion permet de rendre compte des divers idiolectes et sous-cultures souvent conflictuelles de l'émigration.

40. P. Ricœur, La critique et la conviction. Entretien avec François Azouvi et Marc de Launay, Paris, Calmann-Levy, 1995 : «Le terme de reconnaissance me paraît beaucoup plus important que celui d'identité, autour duquel le débat du multiculturalisme tourne la plupart du temps. Dans la notion d'identité, il y a seulement l'idée du même; tandis que la reconnaissance est un concept qui intègre directement l'altérité, qui permet une dialectique du même et de l'autre. La revendication d'identité a toujours quelque chose de violent à l'égard d'autrui. Au contraire, la recherche de la reconnaissance implique la réciprocité. » 


\section{RÉSUMÉS}

Un texte d'ethnopsychiatrie de Tobie Nathan, L'influence qui guérit, permet de souligner quelques opérations rhétoriques relevant globalement d'une interprétation "sauvage ", qui dépend non de la cohérence des données mais de l'arbitraire de l'interprète. Ces diverses procédures - mise en équivalence, durcissement des métaphores, transposition hâtive des termes, faiblesse des inférences, univocité des causes et des interprétations - constituent un système de «mésinterprétation». Au-delà de ces aspects sémantiques, cette problématique, construite autour de la notion d'identité, débouche sur un programme sanitaire et sociopolitique valorisant diverses formes de prises en charge «traditionnelles » des patients et appelant à la création de ghettos culturels. L'article suggère que la prise en compte de la complexité des interprétations autochtones de la déviance, des déterminants sociaux de la maladie, des dynamiques sociales et l'utilisation de concepts comme celui de « reconnaissance » permettraient, à travers les diverses réalités de l'émigration, une meilleure prise en charge des patients.

An ethnopsychiatric text of Tobie Nathan, The influence which cures, allows us to underline some rhetorical operations arising mainly from "wild interpretation", or in other words, from an interpretation which does not depend upon the coherence of facts but upon the arbitrariness of the interpreter. These various procedures - equally matched, hardening of metaphors, hasty translation of terms, weakness of conclusions, univocity of causes and interpretations constitute a system of "misinterpretation". These questions are not however limited to these semantic aspects. Indeed, this problematic, constructed around the notion of identity, opens on to a medical and sociopolitical programme valorizing different forms of "traditional" care of patients and calling for the creation of cultural ghettos. The article suggests that the consideration of the complexity of indigeneous interpretations of deviance, of social determinants of illness, of social dynamics and the utilisation of concepts like "recognition" would allow, besides a better comprehension of different realities of emigration, a better care of patients. 